\title{
RÉFLEXION SUR LA FORMATION ET L'ÉVOLUTION DE LA NORME
}

\author{
OLGA OZOLINA \\ Université de Lettonie, Lettonie \\ PETERIS VANAGS \\ Université de Lettonie, Lettonie
}

\begin{abstract}
Résumé. Il est incontestable que la connaissance des lois historiques du fonctionnement et de l'évolution de la norme linguistique contribue à découvrir le mécanisme des processus linguistiques qui sont à la base de toute langue, déterminent la dynamique de son évolution et participent à la réalisation des tâches actuelles dans le domaine de la planification, la normalisation et la codification linguistique. En tenant compte de ce qui vient d'être constaté et en nous basant sur des recherches réalisées au cours des dernières années dans le domaine de la formation de la norme et de l'évolution des langues, nous tenons à montrer des approches différentes de la norme ainsi que l'état des analyses réalisées ayant trait aux problèmes mentionnés ci-dessus qui restent encore en suspens dans la linguistique romane.
\end{abstract}

Mots-clés : norme, formation, fonctionnement, évolution, variantes, planification, normalisation, codification linguistique

\section{INTRODUCTION}

L'intérêt des linguistes à l'égard de la norme ne s'est jamais épuisé. Certains aspects ayant trait à la notion de la norme sont relativement bien étudiés, notamment l'approche dialectique de la norme, la reconnaissance de sa stabilité d'une part et la tendance à la modification de l'autre part, ainsi que son caractère conventionnel. Il existe néanmoins jusqu’à présent quelques aspects de la norme qui doivent être éclaircis.

Parmi les problèmes à analyser il faut mentionner tout d'abord ceux qui sont liés à la spécificité des niveaux de langue. Il n’est pas non plus suffisamment étudié la question de la corrélation entre la norme, le système de langue, et son usage. Il reste aussi à découvrir les types possibles de norme linguistique et à définir les critères de la normalisation des langues. Il y a également peu de recherches consacrées à la formation et à l'évolution de la norme, à l'étude des principes, 
critères et sources de la norme des langues différentes, sans quoi cependant il est impossible de comprendre à fond la norme d'aujourd'hui.

Il est incontestable que la connaissance des lois historiques du fonctionnement et de l'évolution de la norme linguistique contribue à découvrir le mécanisme des processus linguistiques qui sont à la base de toute langue, déterminent la dynamique de son évolution et participent à la réalisation des tâches actuelles dans le domaine de la planification, la normalisation et la codification linguistique.

En nous basant sur des recherches réalisées à partir de la fin du XXe siècle dans le domaine de la formation de la norme de la langue française et de l'évolution des langues, nous tenons dans cette étude à montrer des approches différentes de la norme ainsi que l'état des analyses réalisées ayant trait aux problèmes.

\section{DIFFÉRENTES APPROCHES DE LA NORME}

On attribue habituellement à la norme des variantes conformément aux niveaux d'analyse linguistique. Dans ce cas, se pose la question s'il s'agit de la langue ou des conceptions de cette langue. Dans le premier cas, il faut parler de la politique linguistique, de la standardisation d'une langue, de sa 'régulation' normative. Dans le deuxième cas, on devrait parler de la norme conformément à la conception théorique de langue. La norme y est attachée à la compétence, à l'idée de la justesse et de la grammaticalité.

Si l'on s'appuie sur les positions de la linguistique communicative, la norme apparaît en tant que phénomène communicatif qui traite la norme comme stabilité élastique. Cette approche de la norme a pour base l'unité de la norme et de sa différenciation qui se trouve en corrélation directe avec la sphère d'emploi de la langue.

Les avantages de cette approche large de la norme sont tout à fait évidents. Elle permet d'unir les aspects différents de la norme : norme comme catégorie purement linguistique, stylistique et culturelle. L'interprétation linguacommunicative de la norme est profondément dialectique. Elle reflète sa vraie nature à double faces: l'unité de l'objectif et du subjectif. Ceci, à son tour, permet de réduire l'axiome linguistique à l'égard de la norme à trois composants principaux : norme objective (comme l'usage accepté par toute la communauté), norme impérative (standard) et norme subjective (usage individuel).

Intéressante et perspective semble l'approche psychosystématique de l'analyse de la norme. Cette théorie a été élaborée par le grand linguiste français Guillaume (1883-1960). L'application de ses conceptions à l'étude théorique de la norme a contribué, à notre avis, à la différenciation plus nette des notions de la norme et de l'usage. Elle a rendu applicable l'évolution de la norme linguistique non seulement à l'enregistrement, mais aussi à la planification scientifique de la langue. 
Selon les psycholinguistes, la norme est une notion à caractère fonctionnel qui se trouve hors du rang langue - parole. A la différence de la triade d'E. Kossériu système - norme - parole, se forme la triade système (langue) - parole (usage) - norme. Si dans la première variante la norme est traitée comme un schéma abstrait, comme un stéréotype général indépendamment des variantes potentielles et réelles d'une langue commune, dans la seconde interprétation la norme est considérée en tant que texte usuel qui a des fonctions de réglementation et qui existe dans l'esprit d'un parlant comme étalon, comme idéal de l'usage. Les fonctions des éléments de la parallèle se partagent de la façon suivante : système (langue) impose, parole (usage) réalise, norme corrige.

\section{TROIS TYPES DE NORME}

Les recherches de l'évolution de la norme dans la langue française amènent à la constatation de l'existence des rapports entre les modifications du système de langue et les changements de la norme. L'évolution de la norme linguistique est un processus de changements graduels des formes d'expression de la norme existante qui se passent conformément aux éléments du système de langue ou bien aux étapes de cette évolution.

Le processus de l'évolution de la norme comprend trois étapes qui correspondent à trois types de norme linguistique (Vlassov, 1986).

1. Norme potentielle qui se caractérise par la possibilité d'avoir des représentations différentes de la parole (usage) et qui correspond à la formation d'un nouvel élément qui marque une étape dans l'évolution du système de langue.

2. Norme optative qui vise à privilégier telle ou telle représentation (usage souhaitable) qui correspond à l'évolution des tendances systémiques.

3. Norme impérative qui cherche à imposer certaines représentations de la parole, mais à en interdire d'autres ce qui correspond à la stabilisation de quelques éléments ou de quelques étapes du système de langue et en même temps permet d'en éviter d'autres.

L'évolution de la norme à la direction de possible, souhaitable, obligatoire correspond la direction de l'évolution du système de langue : potentielle (pluralité de la nomination), tendance (privilège qu'on donne à telle ou telle puissance de la différenciation, tendance à la différenciation, stabilisation de la différenciation (forme), résultat (stabilisation d'une forme parmi d'autres).

Autrement dit, l'évolution de la norme de l'emploi des unités de la langue française dans la direction: norme potentielle, norme optative, norme impérative correspond aux étapes de la différenciation structurale des unités de langue (Ozolina, 2006a : 185-193).

Ainsi l'obligation de la norme n'est en réalité qu'un type d'expression de la norme, notamment de la norme impérative qui constitue une étape finale de la systématisation de la langue, la stabilisation des tendances systémiques tandis que la 
variabilité représente les normes optative et potentielle qui se trouvent au début du processus de la stabilisation de la langue, à l'étape de la formation du système de la langue.

Voilà pourquoi chaque fois en définissant l'emploi correcte ou incorrecte des différentes formes linguistiques, il ne serait pas juste de l'attribuer seulement à la norme codifiée parce que cela pourrait enfreindre le développement de la langue. L'évolution de la norme se réalise surtout grâce aux variantes (Edlička, 1967 : 553).

\section{L'ÉVOLUTION DE LA NORME}

La diversité des étapes de l'évolution caractérise toutes les langues littéraires. Par conséquent, la variabilité de telle ou telle catégorie ne doit pas être considérée comme un défaut, mais comme une forme de son existence. Ce fait amène certains linguistes français à affirmer que la norme n'est pas un code de parler et d'écrire correctement. Ils refusent toutes sortes d'interdictions normatives sous prétexte que d'habitude la langue admet plusieurs variantes d'expression ayant le même contenu. D'après eux, interdire quelques formes d'expression c'est priver un individu du droit de faire son choix (Marsellesi, 1980 : 52).

Les différences qui sont propres au français parlé et écrit prouvent que ceux qui critiquent l'approche la plus répandue de la notion de la norme ont tout à fait raison. La langue parlée d'un Français instruit se distingue nettement de sa langue écrite. Ce qui est considéré comme faute dans la langue écrite est souvent facilement admis dans la langue parlée.

L'attribution à la norme d'un caractère obligatoire ainsi que l'opposition de la norme et de la variabilité conduisent souvent à la négation de l'existence de la norme ou bien à la formation insuffisante de la norme pendant telle ou telle période historique. En même temps les recherches des dernières années font preuve du contraire : la codification fait partie de toute langue et constitue son indice constant sans lequel la langue ne saurait accomplir sa fonction essentielle - être le moyen de communication, de compréhension réciproque et d'actions communes (Volkova, 1983 : 23-26 ; Kassatkin, 1976: 4 ; Kouzmina, 1987 ; Freimane, 1993). Cependant, chaque période historique possède ses propres particularités de la normalisation et de la codification. Il faut distinguer deux périodes, prénationale et nationale. La période prénationale se caractérise par plus grande diversité de formes que la période nationale. Elle possède des variantes chronologiques, sociales, territoriales, fonctionnelles, stylistiques. Il est à noter qu'à cette période le nombre de textes n'est pas grand. L'époque où les imprimeries n'existent pas encore, où les livres ne sont pas édités et la diffusion générale des textes manque, contribue à la diversité des formes d'expression. Ce fait ne signifie pas, toutefois, que les langues prénationales n'ont pas de standard.

Les langues au début de leur évolution sont moins normalisées que les langues littéraires d'aujourd'hui (Boudagov, 1961: 6). Les langues littéraires 
contemporaines se réalisent à travers les textes écrits et oraux appartenant aux styles fonctionnaux différents et fonctionnent grâce à un vaste réseau de massmédias.

Pourtant toutes les langues littéraires d'aujourd'hui ne sont pas strictement normalisées. Par exemple, la langue rhéto-romane littéraire se caractérise par une extrême variabilité à cause de quoi elle ne possède pas de norme d'usage stable (Borodina, $1964: 4$ ).

Ainsi on peut conclure que la norme est une notion historique qui change au cours de la vie de la langue. De plus, le processus de la normalisation d'une langue comprend deux principes de sélection, spontanée (langues anciennes) et consciente (langues récentes) dans leur unité (Subačius, 2002: 131-150). La sélection spontanée devance la sélection consciente et, par conséquent, domine quantitativement au début du fonctionnement de toute langue.

Autrement dit, au cours de la période prénationale prévaut la norme potentielle (la pluralité de la nomination) qui ne vise pas à conserver le système de la langue en usage, mais plutôt à le modifier (Vlassov, 1986). C'est pourquoi l'existence d'un standard linguistique qui admet une grande diversité de formes ne fait pas seulement et uniquement preuve de la normalisation, mais plutôt relève la tendance des textes littéraires de moyen âge à cette normalisation (Volkova, 1983 : 26 ; Ozolina, 2006b ; 267-273 ; Vanags, 2013a : 177-203 ; Vanags, 2013 b : 201-240 ; Vanags, 2019 : 55-86). Il est à noter que la norme potentielle se réalisant spontanément ne se soumet pas à la planification scientifique (Vlassov, 1986).

\section{PLANIFICATION}

Il faut remarquer que lors de la planification scientifique il faut tout d'abord prendre en considération la période concrète de l'évolution de la langue avec tous ses rapports existants entre tous les types de norme qui lui sont propres. Les linguistes doivent participer activement à la formation de l'usage linguistique dans la société en s'appuyant sur les tâches générales et particulières de la planification socio-culturelle. La modification et la planification consciente du type de norme linguistique qui se base sur la prise en considération des besoins de l'évolution sociale et culturelle, forme, à notre avis, l'un des devoirs le plus important dans le domaine de la politique linguistique.

Cependant, l'application de la norme à la politique linguistique suscite elle aussi bien des problèmes. Par rapport à la variation linguistique on distingue deux types de norme : norme objective et norme évaluative (Klinkenberg, 1999: 49-53). La première est mise en évidence par des enquêtes rigoureuses (exemple : $\mathrm{x} \%$ de /ne/ sont omis par les présentatrices de la télévision). La seconde est produite par une attitude sociale, celle qui consiste à étalonner les variétés sur une échelle de légitimité. Une variété légitime est celle que l'on juge acceptable par le corps social (par ex., une négation correctement exprimée comporte un /ne/ ; il est élégant de dire /friterie/ pour /friture/). C'est le choix d'une variété qui constitue le monopole de la légitimité qui, dès lors, est érigée en norme (évaluative). 
Il faut remarquer que la variété standard, dans ce cas, se comforte par des institutions, ce qui fait que les critères de sa sélection sont utilisés d'une manière idéologique. Dans tous les cas, on sélectionne une variété parmi d'autres par des raisons n'ayant rien de linguistique, et on l'impose à l'ensemble du corps social. Par conséquent, on assure le pouvoir symbolique d'une fraction de ce corps social. La distinction entre variétés linguistiques joue aussi un rôle important dans le phénomène plus général de la distinction sociale.

Laction menée par les individus prend souvent la forme du purisme. Il est à noter que l'esprit puriste peut profondément modeler les représentations que le grand public se fait de la langue. Ces représentations peuvent ; à leur tour, avoir des répercussions non négligeables sur le destin d'une langue.

D’après la théorie de la différence du sociolinguiste William Labov (Labov, 1978), chaque groupe social secrète ses propres normes linguistiques par rapport auxquelles se situent ses membres. Ce phénomène est valable aussi bien aux groupes socialement dominants quaux groupes collectivement défavorisés. Les acteurs les plus légitimes du groupe sont ceux qui se conforment le plus strictement à ces normes. Par contre, ceux qui se rapprochent d'une norme extérieure au groupe, fût-elle le bon usage, font figure de marginaux et peuvent même en être exclus.

\section{CONCLUSION}

La spécialisation et puis la légitimation des normes (par ex., le parler des banlieues) renforcent le cloisonnement et la dualisation sociale et contribuent à la création de soi-disant ghettos. Une politique linguistique dans ces conditions, doit-elle naviguer entre les deux extrêmes: d'un côté, valoriser les modes d'expression de l'exclu, de l'autre, combattre la ghettoïsation de la société ? Sans aucun doute, il faut ouvrir l'éventail langagier, mais cesser de courir après le fantôme de la maîtrise totale de la langue (Klinkenberg, 2001 : 41-42).

La norme suscite également beaucoup de problèmes dans le domaine de l'enseignement/apprentissage des langues. Qu'est-ce que connaître ou posséder une langue ? Nous répondons à cette question en nous référant au linguiste belge JeanMarie Klinkenberg (2001 : 43-44) :

C'est cesser de montrer la langue comme un édifice construit d'un seul bloc, mais substituer à cette image celle d'un étal offrant des types linguistiques parmi lesquels on ne saurait choisir au hasard. C'est montrer comment ces variétés se répartissent selon les niveaux économiques, sociaux et culturels, comment elles peuvent ou non être adéquates à une situation donnée, comment elles jouent dans le mécanisme de la distinction. 


\section{RÉFÉRENCES}

Borodina, M. (1964) Sovrémenniy litératourniy rétoromanskiy yazik Chveytzarii. Léningrad : Léningradskaya Akadémia Naouk.

Boudagov, R. (1961) Problémi izoutchénia romanskih litératournih yazikov. Moskva : Vischaya Chkola.

Edlička, A. (1967) O prajskoy téorii litératournogo yazika. Moskva : Prajskiy lingvistitcheskiy kroujok.

Freimane, I. (1993) Valodas kultūra teorētiskā skatījumā. Rīga: Zvaigzne.

Kassatkin, A. (1976) Otcherki istorii litératournogo italianskogo yazika XVIII-XX vékov. Léningrad : Léningradskiy Universitet.

Klinkenberg, J.-M. (1999) Des langues romanes. Paris, Bruxelles : Duculot.

Klinkenberg, J.-M. (2001) La langue et le citoyen. Paris : PUF.

Kouzmina, N. (1987) Stanovlénié normi v oupotréblénii glagolnih imen vo frantzouzskom yaziké XVI véka, Avtoréférat dis. kand. filol. naouk. Léningrad : Léningradskiy Universitet.

Labov, W. (1978) Le parler ordinaire. Paris : Edition de Minuit.

Marsellesi, J.- B. (1980) Langage et processus sociaux. Paris : Edition Larousse.

Ozolina, O. (2006a) Les particularité fonctionnelles et sémantiques des structures attributives des XIe-XIIIe et XXe siècles. Littérature et Linguistique: Diachronie et synchronie. Autour des travaux de Michèle Perret. Sous la direction de Dominique Lignereux (pp. 185-193), [CDROM]. Université de Savoie.

Ozolina, O. (2006b) La variation des moyens d'expression dans l'ancien français. In Actes du XXIVe Congrès International de Linguistique et de Philologie Romanes, Vol. 2 (pp. 267-273). Tübingen : Niemeyer.

Subačius, G. (2002) Two Types of Standard Language History in Europe. Res Balticae, $8: 131-150$.

Vanags, P. (2013a) Latviešu literāāās valodas attīstība. In J. Stradiņš (galv. red.), I. Jansone, A. Vasks (atb. red.) Latvieši un Latvija. Akadēmiskie raksti. I sēj. (pp. 177-203). Rīga: Latvijas Zinātṇu akadēmija.

Vanags, P. (2013b) Latviešu rakstu valodas izveide un attīstība 16.-18. gadsimtā. In A. Veisbergs (red.) Latviešu valoda (pp. 201-240). Rìga: LU Akadēmiskais apgāds.

Vanags, P. (2019) Latviešu valoda pirms Latvijas valsts. In A. Veisbergs (red.) Valoda un valsts. Valsts Valodas Komisija. Raksti, 10. sēj. (pp. 55-86). Apgāds 'Zinātne'.

Vlassov, S. (1986) Evolutzia norm frantzouzskogo sintaksisa. Avtoréférat dis. kand. filol. naouk, Léningrad: Léningradskiy Universitet.

Volkova, Z. (1983) Istoki frantzouzskogo litératournogo yazika. Moskva : Vischaya Chkola.

\section{REFLECTION ON THE FORMATION AND DEVELOPMENT OF THE STANDARD}

Abstract. The knowledge of historical laws of the functioning and development of the linguistic standard is indisputable in the context of discovering the mechanisms of the linguistic processes which are at the very base of any language, determine the dynamics of its evolution and take part in the realization of tasks in the field of planning, standardization and linguistic codification. Taking in consideration what has already been observed and based on the research carried out in the recent years in the field of formation, standard and development of 
languages, the study shows different approaches to the standard as well as the actual state of realised researches which have dealt with the above-mentioned problems that still remain unresolved in the Romance linguistics.

Key words: standard, formation, functioning, development, variety, planning, standardization, linguistic codification

Olga Ozolina $(\mathrm{PhD})$ est professeur associé du Département des Langues Romanes de l'Université de Lettonie à la Faculté des Sciences Humaines. Ses intérêts de recherche portent sur l'histoire des langues romanes, l'histoire de la langue française, la grammaire théorique, la terminologie, les recherches comparatives, la francophonie, la sociolinguistique et la didactique des langues étrangères. Courriel: olga.ozolina@lu.lv

Peteris Vanags (Dr. habil. philol.) professeur de l'Université de Lettonie de la Faculté des Sciences Humaines, Le directeur des programmes d'étude de doctorat. Ses intérêts de recherche portent sur l'histoire des langues baltes. Courriel: peteris.vanags@lu.lv 